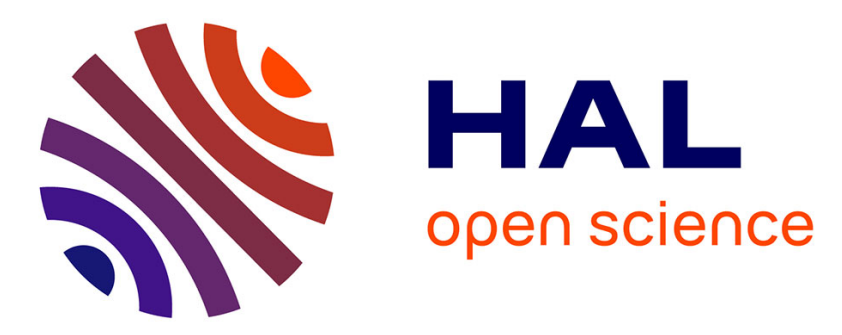

\title{
Implementation of a Wireless Sensor Network Designed to Be Embedded in Reinforced Concrete
}

\author{
Gael Loubet, Alexandru Takacs, Daniela Dragomirescu
}

\section{To cite this version:}

Gael Loubet, Alexandru Takacs, Daniela Dragomirescu. Implementation of a Wireless Sensor Network Designed to Be Embedded in Reinforced Concrete. 46th Annual Conference of the IEEE Industrial Electronics Society (IECON 2020), Oct 2020, Singapore, Singapore. pp.2195-2200, 10.1109/IECON43393.2020.9255268 . hal-03014102

\author{
HAL Id: hal-03014102 \\ https://hal.laas.fr/hal-03014102
}

Submitted on 19 Nov 2020

HAL is a multi-disciplinary open access archive for the deposit and dissemination of scientific research documents, whether they are published or not. The documents may come from teaching and research institutions in France or abroad, or from public or private research centers.
L'archive ouverte pluridisciplinaire HAL, est destinée au dépôt et à la diffusion de documents scientifiques de niveau recherche, publiés ou non, émanant des établissements d'enseignement et de recherche français ou étrangers, des laboratoires publics ou privés. 


\section{Implementation of a Wireless Sensor Network Designed to Be Embedded in Reinforced Concrete.}

\author{
Gaël LOUBET \\ LAAS-CNRS \\ Université de Toulouse, CNRS \\ Toulouse, FRANCE \\ gael.loubet@laas.fr \\ 0000-0003-3347-0036
}

\author{
Alexandru TAKACS \\ LAAS-CNRS, UPS \\ Université de Toulouse, CNRS \\ Toulouse, FRANCE \\ alexandru.takacs@laas.fr
}

\author{
Daniela DRAGOMIRESCU \\ LAAS-CNRS, INSA \\ Université de Toulouse, CNRS \\ Toulouse, FRANCE \\ daniela.dragomirescu@laas.fr \\ 0000-0001-8589-6093
}

\begin{abstract}
This paper presents the McBIM project (Material communicating with the Building Information Modelling) which aspires to provide a communicating reinforced concretes, thus, addresses the design and an implementation of a wireless sensor network devoted to be embedded in reinforced concrete in order to assure structural health monitoring tasks during its entire lifetime. This wireless sensor network is composed of communicating nodes -collecting, processing and sharing with other communicating materials and with virtual models the generated data, and wirelessly powering and controlling the sensing nodes- and sensing nodes -measuring their internal or environmental parameters, pre-processing and transmitting these to the communicating nodes-. The sensing nodes are battery-free and wirelessly powered by a far-field (radiative) wireless power transmission system. Experiments in the air and from a reinforced concrete beam provide encouraging results.
\end{abstract}

Keywords-Wireless Power Transmission (WPT), Wireless Sensor Networks (WSN), Cyber-Physical Systems (CPS), Internet of Things (IoT), Simultaneous Wireless Information and Power Transmission (SWIPT), Communicating Materials, Structural Health Monitoring (SHM).

\section{INTRODUCTION}

With the rise of technologies dedicated to the Internet of Things (IoT), the Wireless Sensor Networks (WSN) are more and more performant, diversified and common. These last can be used in order to implement Cyber-Physical Systems (CPS) to monitor and/or command some physical objects. This is especially the case for Structural Health Monitoring (SHM) applications, for instance of concrete civil structures [1-2]. Moreover, and in order to make these systems fully wireless and offer an expanded lifespan, ambient Energy Harvesting (EH) (e.g. photovoltaic, thermal, mechanical, etc.) and Wireless Power Transmission (WPT) solutions are investigated and developed [3-4]. In this context, the McBIM project (Material communicating with the Building Information Modelling (BIM)) -funded by the French National Research Agency (ANR)- tends to propose an implementation of the concept of communicating materials in the construction domain and more specifically by developing a communicating reinforced concrete [5]. This one must be intrinsically able to generate, store -locally and remotely-, process and exchange data -from its own health and/or environment- with other communicating components and with virtual models -as BIM- through the Internet.

Subsequently, the presentation of the McBIM project will be more detailed. Then, the WSN hardware design developed for the implementation of a communicating concrete will be presented, as well as the experimental results achieved to date and providing a full proof-of-concept. Before to conclude, some critical analysis will be proposed in order to explicit current and future works and the contributions of this research.

This research was funded by the French National Research Agency (ANR) under the McBIM project (Material communicating with the Building Information Modelling), grant number ANR-17-CE10-0014.

\section{THE MCBIM PROJECT}

In order to provide a communicating reinforced concrete linked to an evolutive BIM, a consortium of three French laboratories and one company was set up. Each one has its own role. The LAAS-CNRS (Toulouse) has to implement the hardware part of the communicating reinforced concrete by developing a devoted WSN -this is the purpose of this paper-. The CRAN (Nancy) works on the data collection protocols in the network of communicating reinforced concrete components in order to expend their lifetime by minimizing their energy consumption and need of maintenance, while ensuring their resilience and assuring their scalability [6]. The LIB (Dijon) trades with the data exchanges and the data integration within BIM platforms by respecting the current standards [7]. Finally, FINAO SAS/360SmartConnect (Transen-Provence) prospects building and civil engineering companies to get various real-life use cases, as well as providing reinforced concrete elements to do experiments [8].

The targeted communicating reinforced concrete must meet some constraints. First of all, it must be useful during all its lifecycle: from its manufacture and storage (in the case of precast elements), to its recycling, through the construction of a complete structure based on a combination of elements, as well as the exploitation of this structure, particularly for SHM applications. Thus, its lifespan can be estimated in terms of decades. Then, the communicating reinforced concrete must be scalable in terms of format (e.g. fluid screeds, precast elements for a wall, a bridge deck, etc.) and evolutive during the time in terms of data (type, quantity and periodicity). Indeed, the need in data is function of the step in the lifecycle. For the manufacture, the hourly monitoring of the temperature and humidity evolution during the curing process can help in the decision to continue or not the works (e.g. to unmould a precast element, to tile a floor, etc.). For the storage or the shipping of precast elements and for the construction of a structure, there are the logistic aspects which are the more important, as the ID of elements, their current owner, their location, their delivery address, in which project they must be used, other elements in their neighbourhood, etc. It is also conceivable to create a precise and real-time plan of a structure during its construction, whilst also continuously sharing information between all the stakeholders by using the data stored locally in the element and/or in the BIM. For the exploitation phase, SHM applications are relevant and diverse: the monitoring of temperature and humidity could give information about maturity; the monitoring of the temperature at each side of a wall could be used to quantify its real thermal resistance and certify standards; the cracks detection could allow the application of preventive treatment to avoid irreversible damages and collapses, etc. Thus, with a unique communicating reinforced concrete, different applications can be processed during the entire lifespan of elements by using specific sensors at specific periodicities. 


\section{DESIGN OF THE WIRELESS SENSOR NETWORK}

With the aim of providing a hardware implementation of a communicating reinforced concrete, the choice of the use of a WSN embedded in reinforced concrete for decades was made. In that way, it become possible to render the reinforced concrete intrinsically able to sense its own properties -or these from its environment-, to process, store locally and exchange this data with other communicating materials and with virtual models through the Internet. It also becomes a full CPS.

\section{A. Architecture of the Wireless Sensor Network}

The designed WSN -presented in Fig. 1- allows to produce, process, store and exchange data from the physical world to the physical world (e.g. other communicating materials, owner, user or other stakeholder devices, etc.) and to the digital world through the Internet (e.g. BIM, owner, user or other stakeholder information systems, etc.).

This WSN is composed of two kinds of smart nodes: the Sensing Nodes (SN) and the Communicating Nodes $(\mathrm{CN})$. The SN make measurements, pre-process the collected data and send them to the $\mathrm{CN}$. The $\mathrm{CN}$ collect the data sent by the $\mathrm{SN}$, process and store them. They exchange this data with other $\mathrm{CN}$ constituting the WSN and with other WSN, and virtual models -as BIM- through the Internet. They are reachable through the devices of the owners, users or other stakeholders. Finally, the CN can wirelessly supply the SN. Each component of communicating reinforced concrete embed a number of SN and CN function of its size and its needs (e.g. kinds of sensor, desired spatial precision, etc.).

LoRa wireless technology and LoRaWAN protocol in the European ISM $868 \mathrm{MHz}$ band were chosen as communication solution between SN and CN [9]. Indeed, it seemed to be a relatively low energy consumption way to get long range unidirectional communication. Moreover, a wideband signal (through Chirp Spread Spectrum (CSS) technique) seemed less sensitive to the noise induced by the reinforced concrete which appears very binding for electromagnetic waves. Nevertheless, less consuming and medium range technologies as IEEE 802.15.1 Bluetooth Low Energy (BLE) [10] or IEEE 1902.1 RuBEE [11] are considered for next developments.

As common ambient energy sources are not usable inside reinforced concrete (i.e. there is no light radiation for photovoltaic, there is locally no sufficient gradient of temperature for thermal solutions, natural vibrations of concrete structure are too low for compact mechanical solutions, ambient radiofrequency (RF) energy is too low for RF EH [4]), the RF WPT seemed to be relevant to have a control way to wirelessly supply the $\mathrm{SN}$ by the $\mathrm{CN}$. To have a greater operating distance, the far-field (radiative) solution was preferred to the near-field (inductive) one. Finally, the choice of the European ISM $868 \mathrm{MHz}$ band was driven by a comprised between antenna size and loses in free space.

By using the same frequency band for the wireless communication and power transmission, an implemented and tested solution for Simultaneous Wireless Information and Power Transmission (SWIPT) paradigm is provided [12].

\section{B. Design of the Communicating Nodes}

The communicating nodes -presented in Fig. 2- must be able to gather, process and store the data sent by the $\mathrm{SN}$, as well as share these with other CN, other WSN, stakeholders and digital models through the Internet to update them. They are the energy suppliers of the SN through far-field RF WPT.

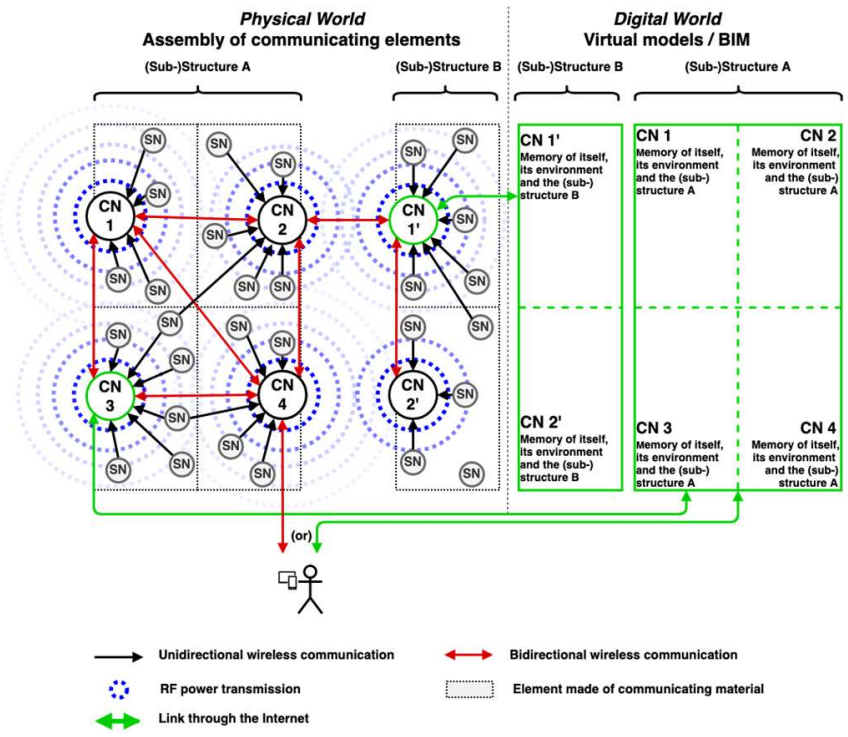

Fig. 1. Bloc diagram of the architecture of the cyber-physical system composing the communicating reinforced concrete.

To achieve these tasks, the current $\mathrm{CN}$ are composed of a LoRaWAN gateway and a RF power source.

The LoRaWAN gateway embeds a LoRa Server and consists of a Raspberry Pi 3 model B+ micro-computer -with a Linux OS- connected to an IMST iC880A LoRaWAN concentrator for European ISM $868 \mathrm{MHz}$ band. Thanks to software, this one collects, processes and stores the data sent by $\mathrm{SN}$ and can access to the Internet and digital world, and be accessed by stakeholders, through an Ethernet or Wi-Fi link.

The RF power source is made of Mini-Circuits components and provides the maximal allowed RF power in the European ISM $868 \mathrm{MHz}$ band of $+33 \mathrm{dBm}$ EIRP (or $2 \mathrm{~W}$ ) [13]. This one allows to wirelessly power the SN.

\section{Design of the Sensing Nodes}

The sensing nodes -presented in Fig. 2- must sense some parameters from their internal state or their environment and wirelessly send the measured data to the $\mathrm{CN}$. The $\mathrm{SN}$ become inaccessible once deployed in the reinforced concrete, thus, these must be reliable, usable for decades by being energy autonomous and battery-free.

The current SN consist of two parts: one dedicated to the power supply and one to the collect and transmission of data.

For the power supply, a rectenna (for rectifying antenna) developed in our team is used to collected RF power transmitted by the $\mathrm{CN}$ and to convert it into electrical power [14]. This dc power is then managed by a Texas Instruments bq25504 Power Management Unit (PMU) which stores it into an AVX BZ01CA223ZSB $22 \mathrm{mF}$ supercapacitor. When enough energy is harvested, the stored energy is used to supply the part dedicated to collect and transmit the data, through a Texas Instruments TPS63031 dc-to-dc buck-boost converter.

For the collect and transmission of the data, a Texas Instruments HDC2010 temperature and humidity sensor achieves the measurement, then the collected data is processed and transmitted by a Murata CMWX1ZZABZ-091 all-in-one LoRaWAN module -which embeds a STMicroelectronics STM32L0 microcontroller and a Semtech SX1276 transceiver- and a meandered monopole antenna. 


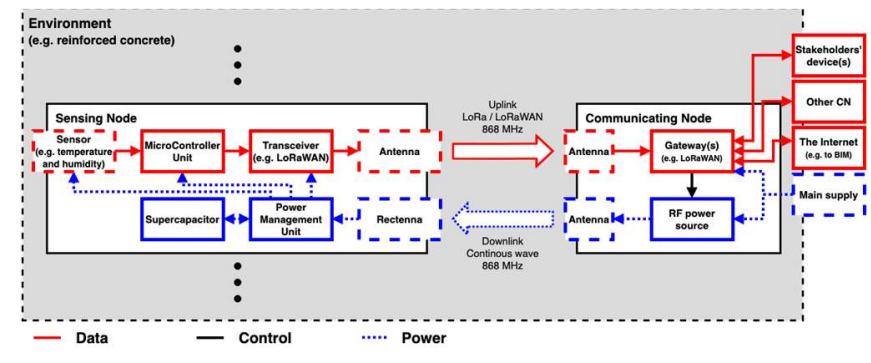

Fig. 2. Bloc diagrams of the sensing nodes and communicating nodes.

\section{EXPERIMENTAL RESULTS}

To certify the proper functioning of our system, several tests -both qualitative and quantitative- have been carried out.

\section{A. Communicating Nodes}

In their current version presented in Fig. 9. C., the communicating nodes are able to receipt the data sent by the $\mathrm{SN}$, as well as process and store it, before make it available to their owner thanks to an Ethernet or Wi-Fi interface, or through the Internet. In addition, they can wirelessly power over several meters many SN located in their neighbourhood.

To provide a Continuous Wave (CW) signal at $868 \mathrm{MHZ}$ with a $+33 \mathrm{dBm}$ EIRP power, the RF power source need nearly $4.45 \mathrm{~W}$ of power with a $12 \mathrm{~V}$ voltage. As well, the LoRaWAN gateway need between $4.0 \mathrm{~W}$ and $4.8 \mathrm{~W}$ of power with a $5 \mathrm{~V}$ voltage to work properly. The amount of needed power is function of its state (standby, processing or reception) and of the connection with other devices (without, through Ethernet or through Wi-Fi). Thus, in the worst case, a 1 Ah battery allows to continuously power the $\mathrm{CN}$ for nearly 45 minutes, and a battery of nearly $32 \mathrm{Ah}$ is needed to power it for $24 \mathrm{~h}$. In order to release constraints during the development phase, the $\mathrm{CN}$ are also accessible and powered by the main supply.

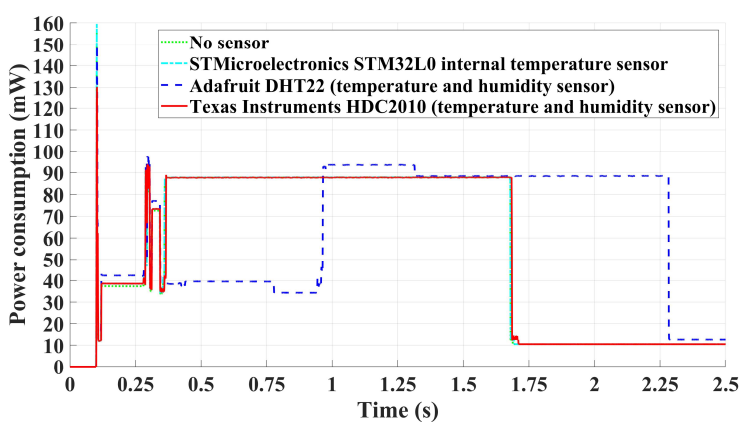

Fig. 3. Consumption of the sensing nodes with various sensors ( $\mathrm{SN}$ is powered at $3.3 \mathrm{~V}$ from Time $=0.1 \mathrm{~s}$ ).

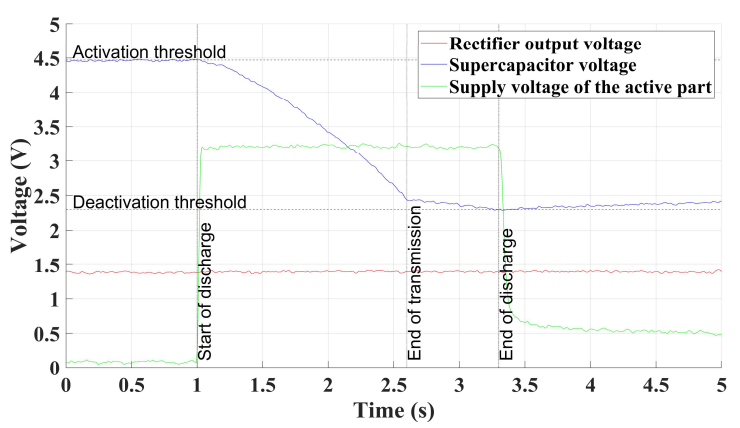

Fig. 4. Use of the stored energy by the sensing nodes with a $+10 \mathrm{dBm} R F$ power available at the input of the rectifier.
Even if the current version of the $\mathrm{CN}$ is composed of two distinct sub-systems: the LoRaWAN gateway and the RF power source; with various useful distances in the air: respectively kilometres and meters; these two elements can be located at the same place without communication losses. To go further, preliminary tests with the use of an Aerotek C111FFF/OPT.N circulator between a monopole antenna (port 1), the LoRaWAN gateway input (port 2) and the RF power source output (port 3) were successfully performed: a $\mathrm{SN}$ at $60 \mathrm{~cm}$ and $160 \mathrm{~cm}$ from the antenna has be wirelessly powered by the $\mathrm{CN}$ and transmitted all its measured data to the $\mathrm{CN}$.

\section{B. Sensing Nodes}

In their current version presented in Fig. 9. B., the sensing nodes allow to monitor temperature and humidity of their near environment and to transmit this data once pre-processed over several kilometres thanks to LoRa technology and LoRaWAN protocol. Furthermore, their energy autonomy is assured by a far-field RF WPT technique which wirelessly powers them over several meters. Besides, the periodicity of measurement and communication can be remotely control through the command of the WPT system.

From the SN presented in [15], several improvements were achieved.

First, a more precise temperature and humidity sensor consuming less time (1.6 $\mathrm{s}$ instead of $2.13 \mathrm{~s}$ for the entire process -measurement and transmission-) and also energy $(130 \mathrm{~mJ}$ instead of $171 \mathrm{~mJ})$ is used as presented in Fig. 3. Also, by using a dc-to-dc buck-boost converter the energy consumption of the active part is reduced too (by limiting the loses due to higher voltages). Thus, even if the capacity could not have been reduced $(22 \mathrm{mF})$, less energy is needed by the $\mathrm{SN}$ (from $214 \mathrm{~mJ}$ to $150 \mathrm{~mJ}$ ) and by reducing the activation (from $5.25 \mathrm{~V}$ to $4.45 \mathrm{~V}$ ) and deactivation (from $2.35 \mathrm{~V}$ to 2.30 V) threshold voltages as shown in Fig. 4, less energy is stored (from $245 \mathrm{~mJ}$ to $159 \mathrm{~mJ}$, always with extra energy), which allows shorter time for recharge.

What is more, different rectifiers were characterized with the $\mathrm{SN}$. A doubler RF rectifier manufactured on $0.8 \mathrm{~mm}$ thick FR4 substrate (Rect1) [14], a compact $(1 \mathrm{~cm} \mathrm{x} 2 \mathrm{~cm})$ serial halfwave RF rectifier manufactured on $1.6 \mathrm{~mm}$ thick FR4 substrate (Rect2) and a serial half-wave RF rectifier manufactured on $0.8 \mathrm{~mm}$ thick Rogers Duroid 5870 substrate (Rect3) [16] -all based on Schottky diodes- were studied and compared for powering the SN. Fig. 5 shows the times needed for each rectifier to achieve a first charge and recharges (in other words, the times needed to have the first measurement and transmission, and between each next ones), as well as their available output voltage, in function of the available RF input power at $868 \mathrm{MHz}$. These rectifiers are characterized at 868 $\mathrm{MHz}$ which is within a few $\mathrm{MHz}$ of their optimum use value. Concerning the output voltages: the Rectl ones are the highest whatever the input RF power, but the Schottky diodes saturate for powers higher than $+6 \mathrm{dBm}$; and the Rect 2 ones are lower than the Rect 3 ones, which is justified by the fact that Rect 2 was designed with size as a priority and Rect 3 with efficiency as priority. Because of this design rule, Rect 2 is less efficient than Rectl and Rect3 with larger first charge and recharge durations. During the cold-start procedure (the main part of the first charge duration), a less than $400 \mathrm{mV}$ voltage is imposed by the PMU, that why Rect3 is faster than Retc1 (which is more efficient for higher voltages) for the first charge. In contrary, when the cold-start procedure is achieved, Rect1 is faster than Rect3 for highest RF input powers, but the 


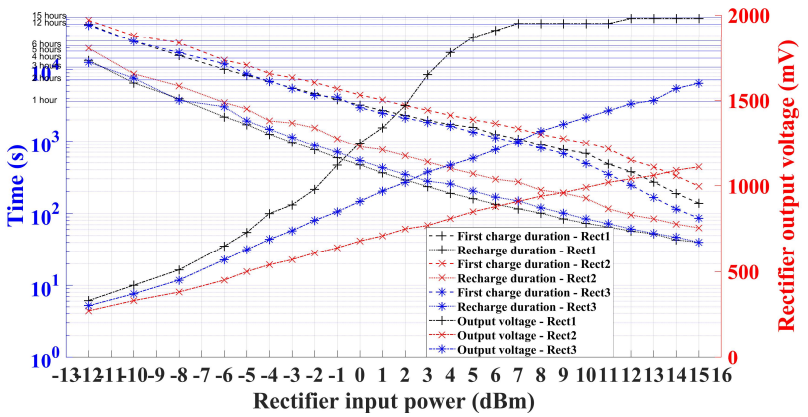

Fig. 5. Durations of the first charge (dashed lines, left) and recharges (dotted lines, left) and rectifier output voltages (dotted and dashed lines, right) as function of the controlled RF power at $868 \mathrm{MHz}$ applied at the input of the rectifier of the sensing nodes for the various rectifiers under test: Rect1 $(+), \operatorname{Rect} 2(\mathrm{x})$ and $\operatorname{Rect} 3(*)$.

two rectifiers are close similar for lowest RF input powers. Regardless of the chosen rectifier, the SN have a periodicity of measurement and transmission which variate between few minutes to hours in function of the available power. Thus, by controlling the RF power source (in terms of transmitted power or duty cycle) it is possible to control wirelessly this periodicity without alter the SN software or hardware parts.

Nevertheless, the energy efficiency of the SN (here defined as the ratio of the energy available at the input of the rectifier (i.e. the integrated available RF input power over the period of interest) to the energy needed for a measurement and transmission) is rather low for the first charge (between 3.4\% and $9.2 \%$ for Rect $1,2.3 \%$ and $7.7 \%$ for Rect 2 and $4.7 \%$ and 9.2\% for Rect 3 ) and recharges (between $13.1 \%$ and $36.4 \%$ for Rect $1,8.3 \%$ and $21.7 \%$ for Rect 2 and $13.1 \%$ and $31.0 \%$ for Rect3), as shown respectively in Fig. 6 and Fig. 7. As said earlier, Rect 3 is more efficient than Rect1 during the first charge whilst this is the opposite during the recharges. For all the rectifiers, the lowest efficiencies during the first charges are for input powers higher than $+4 \mathrm{dBm}$. Only Rect 3 sees this efficiency re-increasing for input powers higher than +12 $\mathrm{dBm}$. Moreover, the lowest efficiencies during the recharges are for lowest and highest input powers. To improve this energy efficiency, the electrical losses and the needs in terms of energy must be limited. For instance, the used supercapacitor has losses up to $11.5 \mu \mathrm{W}$ for a $2.30 \mathrm{~V}$ voltage and up to $22.3 \mu \mathrm{W}$ for a $4.45 \mathrm{~V}$ voltage. As well, the needed energy by the SN can be minimized by optimizing the software to shorten process time, by choosing low consumption sensors and transceivers, etc. In this way, the capacity could be reduced, as well as activation and deactivation threshold voltage, and so the losses could be again limited. Also, developing specific and well-defined components could improve the efficiency by reducing the needed energy: for instance, the PMU used needs at least 15 $\mu \mathrm{W}$ just to work. Last but not least, the development of high efficiency rectifier (or RF-to-dc converter) has a large impact on the energy efficiency. In spite of these weak efficiencies, the SN are usable thanks to the data that can be generated and transmitted periodically, nevertheless this criterium has to be take in consideration to provide a sustainable system.

The PCB $(14 \mathrm{~cm} \times 9 \mathrm{~cm})$ dedicated to the $\mathrm{SN}$ was designed in order to be used as a reflector plan for the rectenna to increase its efficiency and the amount of harvested energy. Even if it was the rectenna presented in [16] (with Rect3) which was first chosen for the SN, the one presented in [14] (with Rect1) was finally privileged because of a higher

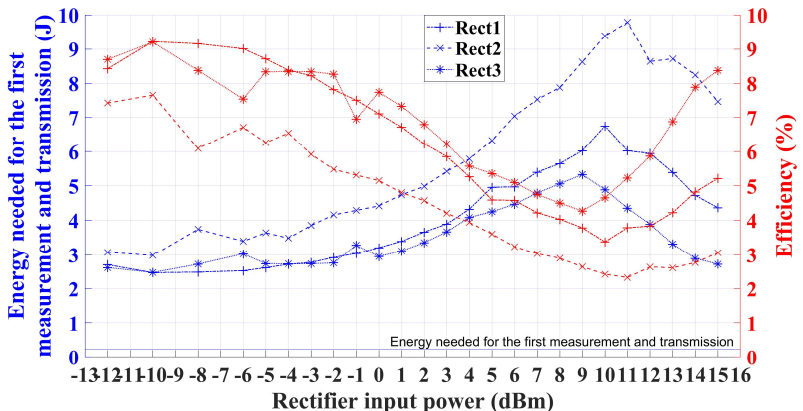

Fig. 6. Energy needed for the first charge (blue) and the energy efficiency of the SN during this first charge (red) as function of the controlled RF power at $868 \mathrm{MHz}$ applied at the input of the rectifier of the sensing nodes for the various rectifiers under test: $\operatorname{Rect} 1(+), \operatorname{Rect} 2(\mathrm{x})$ and $\operatorname{Rect} 3(*)$.

efficiency and compactness (from $11 \mathrm{~cm} \times 6 \mathrm{~cm} \times 3 \mathrm{~cm}$ to 6 $\mathrm{cm} \times 3.5 \mathrm{~cm} \times 1 \mathrm{~cm}$ ), despite a lower gain of the antenna (from $+6.6 \mathrm{dBi}$ to $+5 \mathrm{dBi})$. This choice will allow to decrease the volume of the SN which is constraint by the antenna and supercapacitor sizes. As presented in Fig. 8, there are good correlations for the duration of the recharges and the output voltage of the rectifier between the SN powered through the rectifier by a controlled RF power and the $\mathrm{SN}$ wirelessly powered in the air which scavenges the RF power transmitted by the $\mathrm{CN}$, in the cases of input powers higher than $+2 \mathrm{dBm}$. For lower powers, there are more differences which can be explained by approximations during experiments and in the estimations of the RF power density illuminating the rectenna, but also by the impedance de-adaptation between the rectifier and the antenna which induces an efficiency of the rectenna different than 1, as well as the multipath effects. For these measurements, the SN was located in the air at a distance between $60 \mathrm{~cm}$ and $300 \mathrm{~cm}$ to the power source and were achieved with a $20 \mathrm{~cm}$ step. In spite of these observations, we estimate that the maximal distance between the $\mathrm{CN}$ and the $\mathrm{SN}$ for the WPT should be higher than 5 meters.

To complement these quantitative tests, several qualitative tests were achieved. A network architecture with a unique $\mathrm{CN}$ and multiple SN was tested in the air in order to certify that several $\mathrm{SN}$ can be powered by the same $\mathrm{CN}$ in a specified area and that the data sent by each $\mathrm{SN}$ is well received and processed by the CN. As presented in Fig. 9. A., a SN embedded in a reinforced concrete beam has successfully be wirelessly powered by a CN located $170 \mathrm{~cm}$ away to which it sent successfully all the measured data. This $170 \mathrm{~cm}$ are composed of $15 \mathrm{~cm}$ of reinforced concrete and $155 \mathrm{~cm}$ of air. A specific air cavity was designed in the reinforced concrete

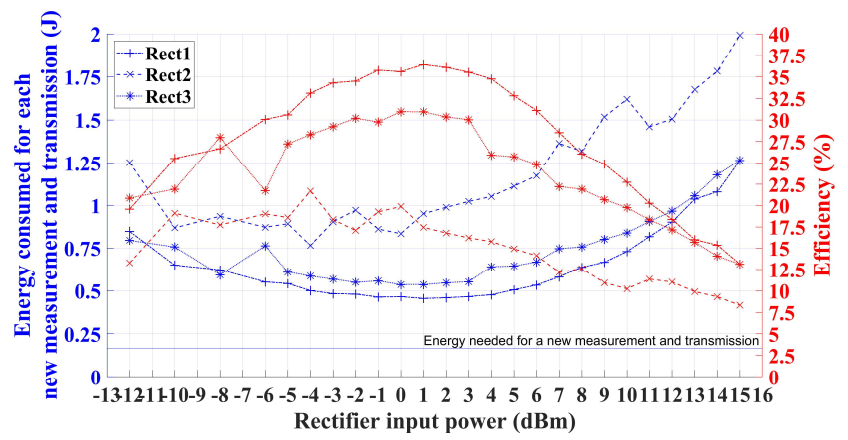

Fig. 7. Energy needed for recharges (blue) and the energy efficiency of the SN during recharges (red) as function of the controlled RF power at $868 \mathrm{MHz}$ applied at the input of the rectifier of the sensing nodes for the various rectifiers under test: Rect1 (+), Rect2 (x) and Rect3 (*). 


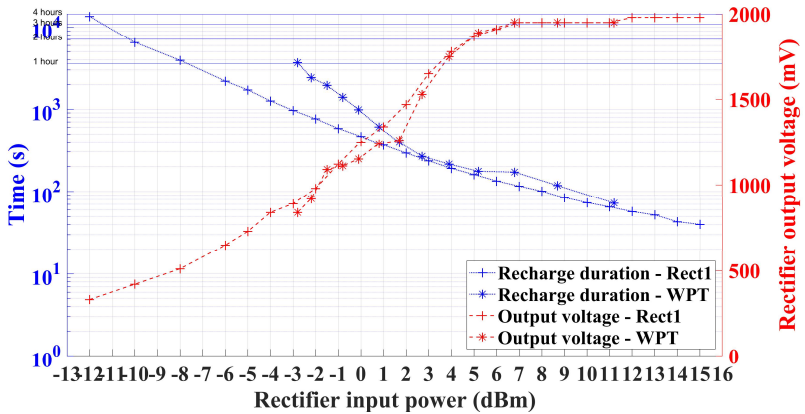

Fig. 8. Duration of the recharges (blue dotted lines) and rectifier output voltages (red dashed lines) as function of the RF power at $868 \mathrm{MHz}$ at the input of the rectifier of the sensing nodes for the single rectifier Rectl $(+)$ and the rectenna $(*)$

beam to achieve these tests which are encouraging with the aim of provided a communicating reinforced concrete. Finally, as for the $\mathrm{CN}$, initial tests using an Aerotek C111FFF/OPT.N circulator between a monopole antenna (port 1), the rectifier input (port 2) and the LoRaWAN transceiver output (port 3) were successfully achieved: a SN at $60 \mathrm{~cm}$ and $160 \mathrm{~cm}$ from the $\mathrm{CN}$ antenna has be wirelessly powered by it and transmitted it all its measured data. In this way, it is possible to use a unique antenna in the $\mathrm{SN}$ and reduce its size.

\section{CRITICAL ANALYSIS}

Even if some results are encouraging in the purpose of implementing a communicating reinforced concrete, more works must still be performed.

\section{A. Work in Progress}

Regarding the sensing nodes, some works are still in progress.

A new version of the $\mathrm{SN}$ with an integrated rectifier is being designed in order to get a more compact and more efficient SN. The integration of a RF circulator is under

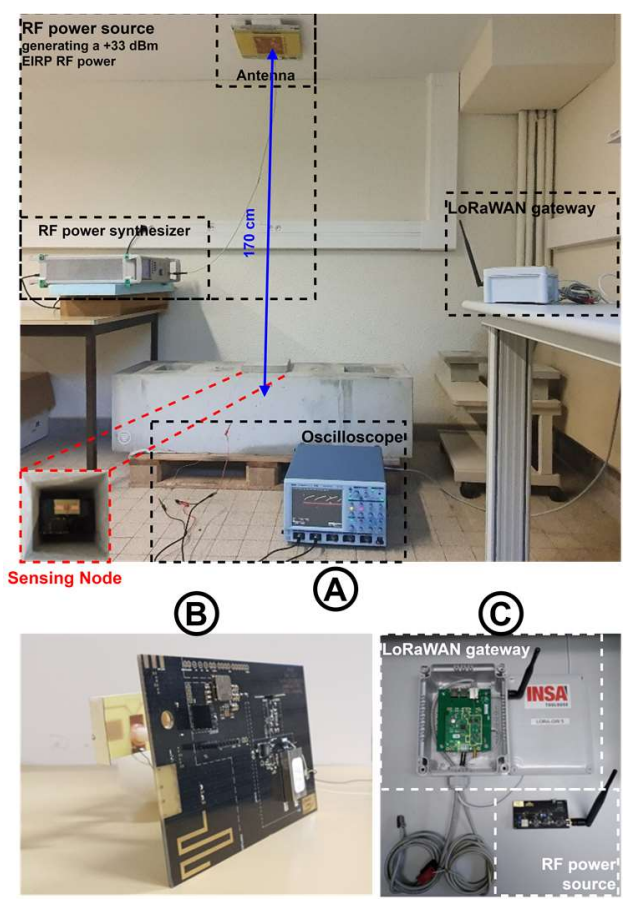

Fig. 9. Photograph of (A) the qualitative test achieved with a sensing node embedded into a reinforced concrete beam, (B) the Sensing Node and (C) the Communicating Node. consideration in the aim to use a unique antenna in the SN. Works on more efficient rectenna are always pursued too.

Lastly, first tests of BLE communication -in master/slaves and broadcasters/listener topologies- into the reinforced concrete beam was performed by using NXP QN9080DK development board. Communication distance of 20 meters can be reached with a board embedded in the reinforced concrete beam even if first packet errors appear from nearly 10 meters. It was also possible to achieve lossless communication between two boards embedded in the reinforced concrete beam at a distance of around $90 \mathrm{~cm}$. Nevertheless, there is no evidence to support that communications took place only in the reinforced concrete, maybe another path (through reinforced concrete and air, with a reflection on the ground) has been followed.

\section{B. Perspectives}

Some other works are planned or seem relevant to pursuit the McBIM project.

Regarding the communicating nodes, the design and manufacture of a one-part and compact version will allow gains in terms of compactness, energy consumption and efficiency. Also, alternatives to the CW RF power source -as pulses RF power source- are studied with the aim to increase the WPT efficiency and decrease the energy consumption.

Regarding the sensing nodes, other kinds of sensor relevant in the case of SHM of reinforced concrete structures -e.g. strain gauges, corrosion sensors, etc.- are searched in order to be integrated in prototypes. More energy efficient components -e.g. supercapacitor, LoRaWAN transceiver, etcare wanted too. SN prototypes with a BLE communication solution could be interesting in order to have the same order of magnitude between the useful distance of WPT and communication, and to provide a solution needing less energy.

\section{Positioning}

In order to ensure the energy autonomy of smart nodes, various strategies can be employed as those proposed in the diverse implementations of battery-free LoRaWAN nodes provided in the past years. Some harvest ambient energies (e.g. solar or thermal [17], mechanical [18], etc.), others are based on backscattering of LoRa signal [19], but although the feasibility of powering LoRaWAN nodes by WPT was demonstrated theoretically in [20], at our best knowledge no full implementation was provided until [15]. By using WPT, the nodes are no more dependent of the availability of ambient energy sources and, by controlling the RF power source, the wireless control of the measurement and transmission periodicity is possible without software or hardware changes.

The majority of the actual wireless and battery-free nodes uses the RFID technologies, which allow short-range communications based on backscattering. These technologies are, for instance, used for SHM applications through active tags [21] or tags intrinsically able to quantify a physical parameter [22]. Nevertheless, implementations based on WPT were proposed too, as in [23] which used short range nearfield (or inductive) WPT and M-BUS medium range communication technology, or in [24] which used medium range far-field WPT and BLE medium range communication technology. Equally, solutions based on RF far-field EH were developed, as in [25] but at the cost of a large system.

Anyhow, at our best knowledges, it does not exist WSN fully embedded into reinforced concrete and composed of 
fully wireless sensing nodes wirelessly powered and controlled by a WPT system, dedicated to the SHM of the material during all its lifetime.

\section{CONCLUSION}

In this paper, the McBIM project has been introduced, as well as the currently obtained results in its scope. Consequently, a wireless sensor network designed in order to be fully embedded into reinforced concrete able to monitor itself during its entire life has been presented. The first prototypes of smart nodes have been characterized and their tests presented. The communicating nodes, composed of a LoRaWAN gateway and a RF power source, are able to receive, process, store and provide to the final user (through the Internet or a direct link) the data collected and transmitted by the sensing nodes. They are able to wirelessly power these sensing nodes and wirelessly control their periodicity of measurement and transmission through a far-field wireless power transmission system. The fully wireless and batteryfree sensing nodes are, themselves, able to sense temperature and humidity, pre-process the measured data and send them wirelessly -via LoRa technology and LoRaWAN protocol- to the communicating nodes. Several experiments in the air and in a reinforced concrete beam suggest that a unique communicating node could power, control and manage tens to hundreds sensing nodes located in an area of few meters around it. A video presenting the functioning of the CPS system implemented in the reinforced concrete will be presented at the conference. Further experiments will be done for reaching the final implementation of a communicating reinforced concrete.

\section{ACKNOWLEDGMENT}

The authors would like to thank Alassane Sidibe and Dr. Abderrahim Okba from LAAS-CNRS (Toulouse, FRANCE) for developing the rectenna; Philippe Hérail from INSA (Toulouse, FRANCE) for his valuable help during BLE experiments; and all their partners in the McBIM project, especially FINAO SAS/360SmartConnect (Trans-enProvence, FRANCE) for providing well-defined use cases and a useful concrete beam for experiments.

\section{REFERENCES}

[1] A.B. Noel, A. Abdaoui, T. Elfouly, M.H. Ahmed, A. Badawy, and M.S. Shehata, "Structural health monitoring using wireless sensor networks: A comprehensive survey," IEEE Commun. Surveys Tuts, vol. 19, no. 3, pp. 1403-1423, April 2017.

[2] M.Z.A. Bhuiyan, J. Wu, G. Wang, J. Cao, W. Jiang, and M. Atiquzzaman, "Towards cyber-physical systems design for structura health monitoring: Hurdles and opportunities," ACM Trans. on CyberPhysical Systems, vol. 1, no. 4, pp. 1-26, October 2017.

[3] H.J. Visser, and R.J. Vullers, "RF energy harvesting and transport for wireless sensor network applications: Principles and requirements," Proc. of the IEEE, vol. 101, no. 6, pp. 1410-1423, April 2013.

[4] J.W. Matiko, N.J. Grabham, S.P. Beeby, and M.J. Tudor, "Review of the application of energy harvesting in buildings," Measurement Science and Technology, vol. 25, no. 1, p. 012002, November 2013.

[5] "McBIM research project," Available online: https://anr.fr/ProjectANR-17-CE10-0014 (accessed on 8 April 2020).

[6] H. Wan, M. David, and W. Derigent, "A holonic manufacturing approach applied to communicate concrete: Concept and first development," International Workshop on Service Orientation in Holonic and Multi-Agent Manufacturing, pp. 414-425, October 2019.

[7] A. Roxin, W. Abdou, D. Ginhac, W. Derigent, D. Dragomirescu, and L. Montegut, "Digital building twins-contributions of the ANR
McBIM project," 15th International Conference on Signal Image Technology \& Internet Based Systems, November 2019.

[8] FINAO SAS, "360SmartConnect," Available online: https://www.360sc.io/ (accessed on 8 April 2020).

[9] LoRa Alliance Technical Committee, "LoRa Alliance technical committee. LoRaWAN 1.1 specification," 2017. Available online: https://lora-alliance.org/sites/default/files/201804/lorawantm specification -v1.1.pdf(accessed on 8 April 2020).

[10] IEEE 802.15.1Working Group, "IEEE standard for information technology-telecommunications and information exchange between systems-local and metropolitan area networks-specific requirementspart 15.1: wireless medium access control (MAC) and physical layer (PHY) specifications for wireless personal area networks (WPANs)," IEEE Standard 802.15.1, 2005.

[11] IEEE 1902.1 Working Group, "IEEE standard for long wavelength wireless network protocol," IEEE Standard 1902.1, 2009.

[12] T.D. Ponnimbaduge Perera, D.N.K. Jayakody, S.K. Sharma, S. Chatzinotas and J. Li, "Simultaneous wireless information and power transfer (SWIPT): Recent advances and future challenges," IEEE Commun. Surveys Tuts, vol. 20, no. 1, pp. 264-302, 2018.

[13] ETSI, "Electromagnetic compatibility and radio spectrum matters (ERM); short range devices (SRD) intended for operation in the bands $865 \mathrm{MHz}$ to $868 \mathrm{MHz}$ and $915 \mathrm{MHz}$ to $921 \mathrm{MHz}$; guidelines for the installation and commissioning of radio frequency identification (RFID) equipment at UHF; ETSI TR 102 436, V2.1.1,” 2014.

[14] A. Sidibe, A. Takacs, G. Loubet, and D. Dragomirescu, "Ultra-compact and high-efficiency rectenna for wireless sensing applications in concrete structure," 2020 IEEE MTT-S International Microwave Symposium, 2020, in press.

[15] G. Loubet, A. Takacs, E. Gardner, A. De Luca, F. Udrea, and D. Dragomirescu, "LoRaWAN battery-free wireless sensors network designed for structural health monitoring in the construction domain," MDPI Sensors, vol. 19, no. 7, p. 1510, January 2019.

[16] A. Okba, A. Takacs, and H. Aubert, "Compact rectennas for ultra-lowpower wireless transmission applications," IEEE Trans. Microw. Theory Tech., vol. 67, no. 5, pp. 1697-1707, March 2019.

[17] W.K. Lee, M.J. Schubert, B.Y. Ooi, and S.J.Q. Ho, "Multi-source energy harvesting and storage for floating wireless sensor network nodes with long range communication capability," IEEE Trans. Ind. Appl., vol. 54, no. 3, pp. 2606-2615, January 2018.

[18] F. Orfei, C.B. Mezzetti, and F. Cottone, "Vibrations powered LoRa sensor: An electromechanical energy harvester working on a real bridge," IEEE Sensors, pp. 1-3, October 2016.

[19] V. Talla, M. Hessar, B. Kellogg, A. Najafi, J.R. Smith, and S. Gollakota, "Lora backscatter: Enabling the vision of ubiquitous connectivity," ACM Trans. on Interactive, Mobile, Wearable and Ubiquitous Technologies, vol. 1, no. 3, pp. 1-24, September 2017.

[20] S. Tjukovs, J. Eidaks, and D. Pikulins., "experimental verification of wireless power transfer ability to sustain the operation of LoRaWAN based wireless sensor node", IEEE Advances in Wireless and Optical Communications, pp.83-88, November, 2018.

[21] S.G.N. Murthy, "Batteryless Wireless RFID based embedded sensors for long term monitoring of reinforced concrete structures," Proc. of the International Symposium Non-Destructive Testing in Civil Engineering, pp. 15-17, September 2015.

[22] J. Zhang, G.Y. Tian, A.M. Marindra, A.I. Sunny, and A.B. Zhao, “A review of passive RFID tag antenna-based sensors and systems for structural health monitoring applications," MDPI Sensors, vol. 17, no. 2, p.265, February 2017

[23] L. Gallucci, C. Menna, L. Angrisani, D. Asprone, R.S.L. Moriello, F. Bonavolontà, and F. Fabbrocino, "An embedded wireless sensor network with wireless power transmission capability for the structural health monitoring of reinforced concrete structures", MDPI Sensors, vol. 17 , no. 11 , p. 2566 , November 2017.

[24] J. Janhunen, K. Mikhaylov, J. Petäjäjärvi, and M. Sonkki, "Wireless energy transfer powered wireless sensor node for green IoT: Design, implementation and evaluation," MDPI Sensors, vol. 19, no. 1, p. 90, January 2019

[25] A.N. Parks, A.P. Sample, Y. Zhao, and J.R. Smith, "A wireless sensing platform utilizing ambient RF energy," IEEE Topical Conference on Biomedical Wireless Technologies, Networks, and Sensing Systems, pp. 154-156, January 2013. 